\title{
SISTEMA DE FLUJOS DE ENERGÍA PARA FACILITAR LAS NEGOCIACIONES DEL CLIMA
}

\author{
Ramiro Valdivia Herrera
}

\section{Resumen}

El Congreso peruano, a nombre de la Unión Interparlamentaria - Grupo Latinoamericano y del Caribe, en su Pronunciamiento en la COP 15 propuso: Un Sistema de Transacciones Forzantes para basar las negociaciones del Clima en unidades de energía $\mathrm{W} / \mathrm{m}^{2}$ en lugar de toneladas de carbón equivalente (para usar el Sistema Internacional de Unidades SI, en lugar de un sistema artificial de carbón); y así incluir todos los forzamientos que afectan el Balance de Radiación Terrestre con el Universo, facilitando la recuperación de los ecosistemas y el Balance de Energía Planetario. El artículo muestra la ciencia básica para la propuesta y explora las consecuencias en caso lo adoptase la CMNUCC.

Palabras Clave: Sistemas de flujos, balance energético, ética ecológica, productividad, eficiencia.

\section{Abstract}

It was proposed by the Peruvian Congress, on behalf of the Inter-Parliamentary Union - Group of Latin American and The Caribbean, in its pronouncement at the COP 15: a Forcing Transaction System in order to establish climate negotiations in energy units $\mathrm{W} / \mathrm{m}^{2}$ instead of tons of coal equivalent (to use The International System of Units - SI, instead of an artificial system of coal); and so include all the forcing that affect the Earth Radiation Budget with the Universe, facilitating the recovery of ecosystems and the Planetary Energy Balance. This article shows basic science for the proposal and explores the consequences if the UNFCC decides to adopt it.

Key words: Systems of flows, power balance, ethical ecological, productivity, efficiency. 


\section{Un sistema de intercambio de energía para negociaciones del clima fáciles.}

La ciencia del clima habla de forzamientos, el término se utiliza también al analizar el equilibrio de la energía de radiación de la tierra con el universo. Un documento anterior vinculado aquí (Río+20Español+Inglés+descarga) muestra porqué es importante evolucionar en las negociaciones del clima desde un sistema de transacción de emisiones (CMNUCC) STE, a un sistema de intercambio de forzantes SIF, donde el primero es un caso particular del segundo, la necesidad de aclarar la condición de nuestra casa la tierra, no sólo de las emisiones de gases de efecto invernadero, sino su acumulado ligado al cambio climático, la acidificación de los océanos, la contaminación pululante, la calidad del agua y su tratamiento, la conservación de la biodiversidad.

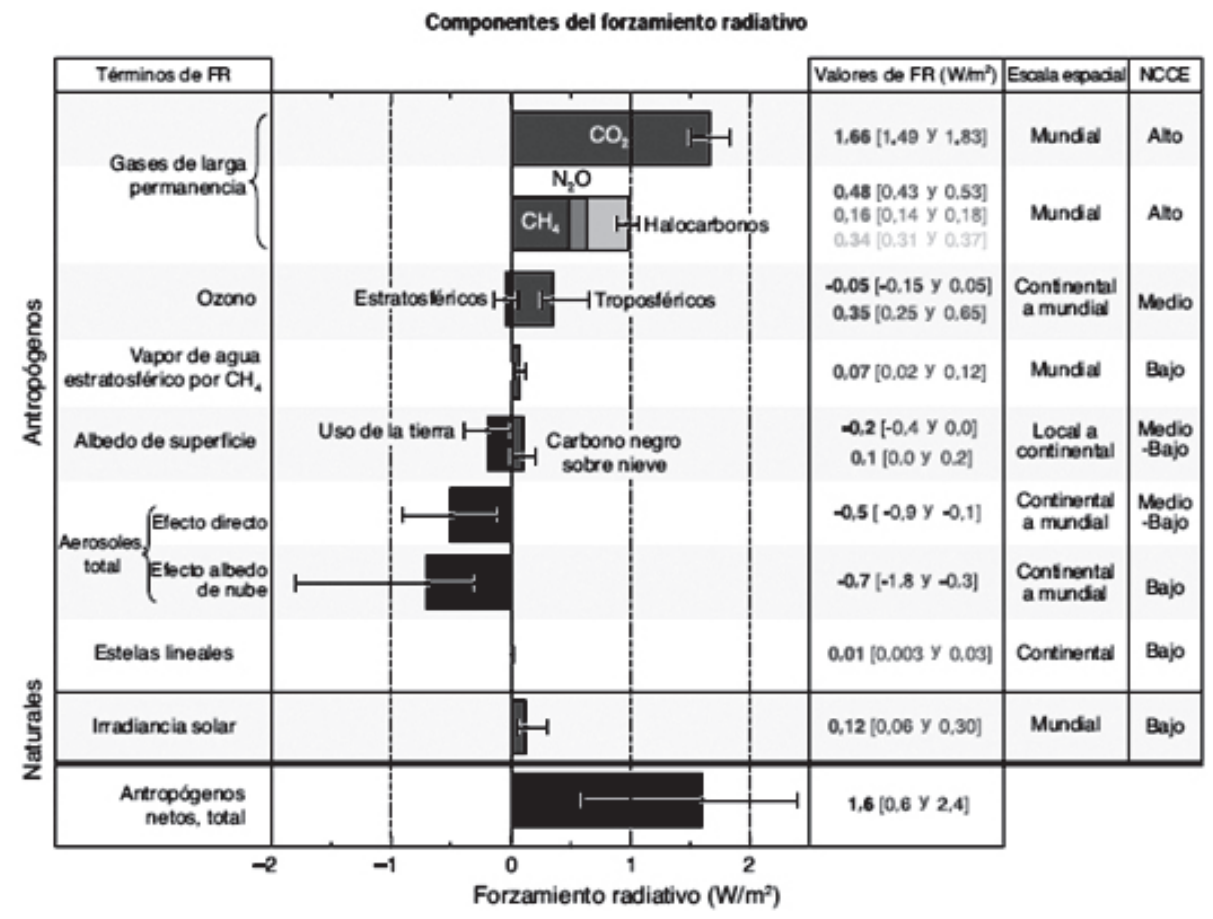

Figura 2. Promodio mundial dd forzaminnto r adiativo (FR) en 2005 (estimnciones cotimas y horauilla do incotidumbres dd 5 al $95 \%$ ) respecto de 1750 para $\mathrm{CO}_{7} \mathrm{CH}_{e}, \mathrm{~N} \mathrm{O}$ y otros agentes y mecanismos importantes, extension geografica opica fescala espaciat) del forzamiento, y nivel de conocimiento cientifico NDCC) evaluada Los aerosoles procedentes de expciones volcanicas explosivas ahaden un armino de enf iamiento episodico derante cierto numero de anos despues de una erupcion. En el intervalo de valores de las estelas de condensacion lineales no se incityan ctros posibles efectes de la aviacion satre la rubosidad. (GII Figura RRP.2)

Un ejemplo: podemos notar que la irradiación media o constante solar $\left(1.361 \mathrm{kw} / \mathrm{m}^{2}\right)$, comparada con el forzamiento $\left(\sim 0.11 \mathrm{Wm}^{2}\right)$ del Sol, muestran 4 órdenes de magnitud de diferencia, y algunos hechos 1) si usted vive en los trópicos puede tener más de un kilovatio local de 
energía por metro cuadrado, provisto gratuitamente por el sol de mediodía, al que se puede exponer con todos los beneficios de la prudencia, o convertir en electricidad, calor, fotoquímica, o simple usar en iluminación. 2) E1 forzamiento relativo del sol comparado al forzamiento de los gases de efecto invernadero $\left(\sim 2 \mathrm{~W} / \mathrm{m}^{2}\right)$ es 20 veces menor, o el efecto antropogénico sobre la atmósfera es 20 veces mayor que cualquier aumento medio de la radiación solar natural. Con el fin de ser claro, se debe mencionar el poco entendimiento del papel que juegan los aerosoles, y que recientemente los cálculos y medidas sobre la tierra y lo profundo de los océanos muestran que el calor extra o el forzamiento total neto tiene un valor medio de flujo de energía positiva de $\sim>0.5 \mathrm{w} / \mathrm{m}^{2}$. No hay que olvidar que si queremos calcular la cantidad de flujo de energía extra hacia la tierra que esto representa al año, por la sección transversal de la tierra, (>pi x el cuadrado de 6,5 millones de metros), le da un valor varias veces mayor que todo el uso anual de energía por la humanidad.

En la CMNUCC, Convención marco de las Naciones Unidas sobre el Cambio Climático, la Unidad de intercambio es la tonelada de $\mathrm{CO} 2$ equivalente, subterránea unidad de energía equivalente al forzamiento de una tonelada de $\mathrm{CO} 2$ en la atmósfera durante 100 años. De otro lado, la unidad de forzamiento es el vatio por metro cuadrado, igual que la de flujo de energía, positivo cuando ingresa o calienta, y negativo cuando sale o enfría. El concepto de forzamiento no solo toma en cuenta los procesos de carbono, sino cualquier acción de parte del ecosistema natural en el balance al nuevo equilibrio de flujo de energía en la tierra. Un químico dijo, sencillo: el balance de energía: de los glaciares, océanos, bosques, desiertos, nubes, todos evaluados en $\mathrm{W} / \mathrm{m}^{2}$ unidades de flujo internacional de energía, realmente describen el estado de base global del clima. (No STE, CMNUCC: la CM Naciones Unidas Financiando la Combustión de Carbono).

La socialización de estos cambios paradigma, de la economía neo liberal de recurso fósil infinito a los límites ecológicos de la energía, fundamentalmente ayuda a comprender el papel abusivo de las energías combustibles fósiles, relacionado con la disponibilidad de energía en cualquier lugar, con la variabilidad del cambio climático $\mathrm{y}$ su sintoma de vulnerabilidad social. Estas consecuencias inconvenientes, por consumo de combustibles con emisión de gases de efecto invernadero, puede declararlos tóxicos para el desarrollo humano sostenible, e impuesto para no subsidiar efectos colaterales en la salud pública, junto al cambio ético social respecto a quemar C- Combustible o no. Las pruebas muestran la existencia abundante de energía limpia, pero las soluciones locales de tecnología no están uniformemente distribuidas en el mundo, y requiere construir las capacidades humanas en cienciatecnología física de la energía, 
fundamentalmente decisión politica para poner en práctica finanzas y políticas públicas favoreciendo el desarrollo local descentralizado limpio.

El principio de incertidumbre de Heisenberg en la naturaleza, relaciona el producto de las incertidumbres en la energía y el tiempo, el que siempre será mayor que una cantidad constante $\mathrm{h}<\Delta . \Delta \mathrm{T}$. Curiosamente, el uso humano de combustibles fósiles, una energía formada hace millones de años quemada hoy, está enviando realmente el estado de la atmósfera terrestre a condiciones experimentadas algunos millones años antes. Literalmente, muestra que según la agenda, la atmósfera con 390ppmCO2 de forzamiento es lo que experimentó aproximadamente la Tierra 0.6 millones de años antes. CMNUCC ha declarado que condiciones de desastre climático generalizado se alcanzarán cuando el tiempo atmosférico equivalente esté de regreso 3,5 millones de años, cuando el contenido de $\mathrm{CO} 2$ en la atmósfera sea $440 \mathrm{ppm}$, o $\Delta^{\circ} \mathrm{T}$ global $>2^{\circ} \mathrm{C}$, con una Sensibilidad del Clima igual a $3^{\circ} \mathrm{C}(\mathrm{al}$ doblamiento de $\mathrm{CO} 2)$. Al ritmo actual de inyección de GEI (3ppmCO2/año), estamos a menos de 20 años para que llegue, a menos que se decida abandonar-cortar- restringir ahora en serio el uso de cualquier combustible con gases de efecto invernadero.

Un SIF, sistema de intercambio de forzantes (Flujos de energía) de la energía de terrestre para mantener el equilibrio, utiliza directamente unidades de energía para transaccionesnegociaciones relacionadas al clima, reconociendo que el cambio climático es una alteración terrestre del tiempo atmosférico, relacionado a la energía limpia (conocimiento, instalaciones, equipo, la cooperación en cienciatecnología) que es la forma de compensar con mayor equidad. En una tierra bajo la presión humana, un sistema de transacción ecológico, midiendo las acciones de energía limpia frente a la de combustible con gases de efecto invernadero, todas en el Sistema Internacional de Unidades $\mathrm{W} / \mathrm{m}^{2}$, son la forma natural de discutir y recuperar el equilibrio de la energía terrestre.

\section{Algunas consecuencias físicas}

La evaluación del efecto que sobre la radiación terrestre ejercen los diferentes sistemas o forzantes en $\mathrm{W} / \mathrm{m}^{2}$, proporciona una medida fundamental en la búsqueda de la estabilización del clima, porque se centra en el nivel de energía basal del planeta en equilibrio con el espacio profundo, en la comprensión que tales cambios en el estado basal, significan cambios en el estado general del clima del planeta.

$\mathbf{F}=\Sigma$ (rst), / $\mathbf{S T}=$ Forzamiento (densidad-flujo $O$ radiación de energia, Superficie, Tiempo)

En tal forma, la operatividad de la Comunicación Nacional (UNFCC) podría mejorarse: como una matriz de acumulados históricos de forzamientos.

$\mathrm{F}=[\mathrm{CN}=\Sigma$ (Toneladas de emisiones: $\mathrm{CO} 2, \mathrm{CH} 4, \mathrm{NO} 2$, zona arbolada, zona de los glaciares, etc.) en producto 
escalar. Matriz Unitaria Forzante Equivalente (forzante unitario1, fu2, fu3, fuj $)=F E] \Sigma F j=(F 1+F 2+F 3+\ldots F j)$ $\mathrm{j}=\mathrm{Fj}=\mathrm{F}$ (nación)j $=\left(\mathrm{CN}\right.$. FE) $\left(\mathrm{W} / \mathrm{m}^{2}\right)$

Así, idealmente, el forzamiento global sería la suma sopesada resultante en superficie de todos los forzamientos nacionales, sobre la superficie de la tierra $\mathrm{Fg}=\Sigma \mathrm{Fj}$. Sj / S la tierra

El producto $\mathrm{Fj}, \mathrm{Sj} . \Delta \mathrm{T}$ durante un intervalo de tiempo arbitrario, que podríamos llamar forzado neto de la nación, puede ayudar a comparar internacionalmente la energía de calefacción acumulada, responsabilidad de cada nación, y el cambio de estado de energía de la tierra y así del clima.

\section{Paréntesis de ética ecológica}

Debido a que el cambio climático actual está vinculado al desequilibrio de la energía de la tierra con el Universo, y (la restauración del equilibrio sólo se dará cuando el Forzamiento de emisiones de $\mathrm{CO} 2$ equivalente se estabilice en el tiempo, a un diferente nivel de equilibrio $\mathrm{T}^{\circ} \mathrm{eq}$ ) el origen de este forzamiento es la ética de la humanidad en lo que respecta a quemar fósiles y obtener energía en vez de tomarla de los alrededores, como la naturaleza lo hace, nuestras acciones no siguen el principio de mínima acción en la naturaleza, por lo que nuestra eco-eficiencia va a menos.

Algunos mitos creados para apoyar el estado real de poder centralizado en combustibles fósiles en la sociedad humana es que la quema de combustibles es una solución con eficacia, pero eso es ahora una mentira pasada. Todo principio biológico puede demostrar que somos una especie ineficiente, que el comportamiento de plaga es nuestro, peor de cáncer, debido a que queda necrótico el propio cuerpo- la tierra. Las pruebas muestran que territorios, ciudades, países sobre poblados es en realidad una ruta perniciosa, y es necesario (para constituir con la tierra una unidad biológica sostenible) cambiar en nuestra vida el nivel de uso ineficiente de la energía.

¿Qué hacer? ¿Cómo? Son preguntas en el aire, cuando es ahora, no cabe duda, que debemos pensar en remediación.

Por ejemplo: se puede explicar a los jóvenes que la vida sin la reproducción en familia puede demostrar inteligencia, y si usted necesita más amor alrededor, la atención de los huérfanos del mundo es humanidad real, ya que urge dejar de crecer como especie en conjunto, ya que hay insuficiente espacio-tiempo (la prueba: estrés de vida en las grandes ciudades).

Todo lo que se usa-compra debe tener un ciclo de vida acordado por la sociedad, donde se prohíba las áreas de disposición de basura, los desechos, orgánicos, compost, metales, pilas, aparatos, equipos, agua, todo tiene que preservar un valor en armonía con nuestro ineludible planeta civilizado.

La actual tasa global de aumento de calentamiento global muestra que el mercado de $\mathrm{CO} 2$ o del carbono 
no pudo pararlo, que crece la deuda ecológica acumulada y que no da buena orientación a ningún ciudadano común para resolver algo que físicamente sólo tiene relación con la energía no con el dinero, pero que en realidad se discute en términos de quién contamina paga, no en términos de que el calor debe enfriar proporcionalmente para compensar. En el balance de la energía de la Tierra con el universo, el flujo de energía o forzamiento cuenta como sistema físico y no el dinero; evitar el dinero (mercado de CO2) simplifica la discusión del clima a términos de energía.

Algunas consecuencias sociológicas de política jurídica si el CMNUCC acepta un SIF como modelo de mesa de negociaciones sobre el clima, -lo que se discutió en un artículo anterior (Río+20)-, destaca ahí la consecuencia cero que el forzamiento de los acumulados de gases de efecto invernadero, la presencia de aerosoles, el retroceso de los glaciares, la acidificación de los océanos, el uso del suelo, cualquier flujo de energía al cambiar un ecosistema cuenta, en una base de acción común y responsable para estabilizar el clima, o restaurar el balance de equilibrio de la energía radiactiva de la tierra con el universo.

Aquí solo se muestran algunas simplificaciones de nomenclatura y aclaración de términos, en la evolución desde un STE a un SIF, en un contexto de negociaciones entre las naciones por el cambio climático de calentamiento global.
1. Desde un sistema de "transacción de emisiones" STE, a un sistema de intercambio de forzamientos, flujos de energía SIF entre las naciones, polos y océanos...

2. Desde la reducción de emisiones para evitar la deforestación y la degradación de las tierras o REDD, a proyectos para preservar los bosques y la cobertura de la tierra.

3. De mecanismos de desarrollo limpio MDL a mecanismos de implementación de energías limpias MIEL.

4. Desde certificación de sustracción de emisiones a proyectos de forestación acumulada.

5. De la certificación de reducción de emisiones a certificados por evitar quema y emisiones.

6. Etc.

En general, un sinceramiento real de las palabras usadas en la discusión que se debe dar entre los países, comunidades, personas, para evitar el cambio climático peligroso, e interpretaciones caprichosas de abogados en los mercados de carbono. Aquí, dos enfoques del concepto de energía aplicados a asuntos sociales ayudan a evitar la degradación de los ecosistemas.

\section{El principio de mínima acción en la naturaleza}

Mecánicamente, expresa el hecho de que a partir de los caminos posibles entre dos puntos, la naturaleza elige uno en que una cantidad llamada 
acción, igual a la diferencia entre la energía cinética menos la energía potencial, integradas en el tiempo y espacio, es la más baja. Esta condición se cumple sólo en acuerdo a las leyes de Newton, y si se le preguntara a la humanidad si se conduce bajo este principio, que regula el flujo del agua, el viento, las corrientes eléctricas y relámpagos, el viaje de luz en fibras ópticas, las comunicaciones láser, el crecimiento de las plantas, sin duda la respuesta es no.

Mediante el análisis de la acción A $=\Sigma(\mathrm{EC}-\mathrm{EP}) \Delta \mathrm{T}, \Delta \mathrm{L}$, podemos establecer límites de integración del tiempo de 1750 a la fecha, y, por lo tanto, encontramos que la energía cinética puesta en marcha, térmica, eléctrica o consumida activamente, ha crecido tanto como 16TW año y se espera que llegue a duplicarse para el 2050; mientras que la energía potencial de todo tipo (por quema de combustibles fósiles, deforestación, acidificación de los océanos, la reducción de la biodiversidad, la retracción de los glaciares) se ha reducido desproporcionalmente. Por lo tanto, aumentar la diferencia entre estos tipos de energía da como resultado que nuestras acciones no tienen nada de natural, o no imitan el principio de mínima acción (eficacia) que gobierna la naturaleza.

En el cálculo de la acción, esta es mínima cuando no hay fricciónconflicto entre las energías en transformación; la suma es también cero si el cambio en el tiempo es igual a cero, lo que equivale a no operar, e indica que a la humanidad le es aconsejable tomar una pausa natural de descanso.

El principio de mínima acción de la naturaleza muestra hoy en día un desequilibrio máximo por la acción antrópica, por lo que es prudente disminuir la energía cinética industrial y aumentar los potenciales de energía natural, o las primeras pueden superar obstáculos, quizás barriendo la humanidad.

\section{Productividad contra eficiencia}

En el paradigma de la cultura participativa de la comunidad rural frente al crecimiento de la productividad individual egoísta, la primera selección apropiada para cambiar la tendencia climática peligrosa, la aceptación del enlace entre las personas y la tierra, hace que la gente rural tienda a armonizar su actuación con la tierra circundante. Como sabemos, el comportamiento humano civilizado tiende a modificar sin tener en cuenta el impacto sobre lo que le rodea. Lecciones drásticas enseñan a no seguir estas pautas de comportamiento, o esperar amplias reacciones desde la naturaleza.

La justificación de la economía tradicional para todo tipo de actos de abuso es el aumento de la productividad = [ cuánto obtengo/ cuanto pongo], concepto que no tiene precisión en absoluto, porque compara situaciones cualitativamente diferentes en un intervalo de tiempo definido (por ejemplo: volumen de cultivo orgánico contra producción agrícola con químicos). Se puede mostrar que en la 
mayoría de situaciones industriales, un aumento de la productividad implica la entrada de energía adicional, y así la eficiencia ecológica = [energía útil/energía de entrada], va a menos. "La productividad cultural" puede aumentar también sin aumento en el consumo de energía fósil, al ir la energía de entrada a menos y subir el cociente de eficiencia, preservando la energía útil.

\section{Conclusión}

Utilizar conceptos del equilibrio de la energia de la Tierra puede facilitar las mesas de negociación internacional sobre cambio climático, pero es necesario evolucionar de un sistema aceptado de transacción de emisiones a un sistema de intercambio de forzantes, de medir comparativamente las acciones de la humanidad sobre los ecosistemas en toneladas de $\mathrm{CO} 2$ equivalentes, a calificar directamente las acciones humanas de calefacción o refrigeración sobre la superficie de la tierra en unidades de flujo de energía $\mathrm{W} / \mathrm{m}^{2}$.

\section{WEBGRAFÍA}

http://www.ipcc.ch

http://www.columbia.edu/-jeh1

http://www.unfcc.int

http://www.qoylluritty.org

http://www.unido.org 